\title{
FOREIGN BODY IN THE URINARY BLADDER OF 14-YEAR-OLD BOY: CASE REPORT AND REVIEW OF LITERATURE
}

\author{
Svjetlana MUJAGIĆ1 ${ }^{1}$ Snežana ZULIĆ , Samed JAGODIĆ 3 \\ ${ }^{1}$ Department of Radiology and Nuclear \\ Medicine, ${ }^{2}$ Department of Pediatrics \\ ${ }^{3}$ Department of Surgery of the University \\ Clinical Center Tuzla, Bosnia and \\ Herzegovina \\ Corresponding author: \\ Svjetlana Mujagić \\ Ahmeta Kobića 19 \\ 75000 Tuzla, \\ Bosnia and Herzegovina \\ svjetlanabh@gmail.com \\ Tel.: + 38735303585 \\ Fax.: + 38535251456 \\ Received: June 17, 2014 \\ Accepted: September 8, 2014

\begin{abstract}
Objective - Foreign bodies (FB) in the urinary bladder (UB) in adults are frequent while in children this condition has been rarely reported in the literature. Case report - A 14-year-old boy was admitted to the hospital because of abdominal pain, dysuria, traces of blood on the underwear and temperature of $38.5 \mathrm{C}^{\circ}$. He denied self-insertion of $\mathrm{FB}$ into the urinary system. Abdominal ultrasound and intravenous urogram showed a tubular structure in the UB, and cystoscopy revealed a thin tube-like structure in the UB, that was partly incorporated in the wall of the bladder, suspicious of FB. Tumour mass such as a teratoma could not be excluded, also. As a result and especially due to the fact that the boy denied self-insertion of the FB, cystoscopic removal of the FB could not be performed. Computed tomography revealed a hypodense area into the UB that spreading through the inflamated wall of the bladder into the left obturator internus muscle. Suprapubic cystostomy was done and ear cleaning stick $10 \mathrm{~cm}$ in lenght was found into the UB. The boy confessed self-insertion of the FB during erotic stimulation. Conclusion - In the differential diagnosis of the causes of unknown genital bleeding and hematuria, one should always think
\end{abstract} \\ of a $\mathrm{FB}$, even when the patient denies self-insertion.
}

Copyright (C) 2015 by

University Clinical Centre Tuzla.

E-mail for permission to publish: paediatricstoday@ukctuzla.ba
Key words: Urinary bladder - Foreign body - Cystoscopy - Intravenous urography - Computed tomography.

\section{Introduction}

FBs in the UB in adults are frequent while in children this condition has been rarely reported in the literature $(1,2)$. The types of FB have been classified as inserted, migratory and iatrogenic (3). Intravesical FBs are urological and radiological challenge in diagnosis and management (4). This condition can cause diagnostic difficulties, especially if there is no information about the trauma, any previous genitourinary medical procedure, or in cases where the patient denies self-insertion of a FB into the urethra and the UB.
In this case report we present a 14 yearold-boy with dysuria in whose UB, after diagnostic procedures and UB surgery, an ear cleaning stick was found which had been selfinserted.

\section{Case report}

A 14-year-old boy was admitted to the Department of Paediatrics because of abdominal pain and dysuria over the previous week; traces of blood on his underwear over the previous three days, and a temperature of 
38.5 Co on the day of admission. The boy, as well as his father, denied previous trauma and genitourinary medical procedures. The boy also denied self-insertion of a FB into the urinary system. Apart from suprapubic pain on palpation, other physical examinations were normal. Laboratory tests revealed: increased sedimentation 52/84 (normal values 12/24), elevated C-reactive protein 53.7 $\mathrm{mg} / \mathrm{l}$ (normal range $0-3.4 \mathrm{mg} / \mathrm{l}$ ), proteinuria and haematuria, while the urine sediment revealed leucocytes in groups and erythrocytes present in abundance. Staphylococcus aureus was isolated from two urine cultures $\left(10^{5}\right.$ bacteria $\left./ \mathrm{ml}\right)$. Creatinine and blood urea were within the normal range. Abdominal ultrasound revealed a hyperechogenic tubular structure in the UB, with locally thickened wall (Fig. 1). Native x-ray of the urinary tract did not show any pathological findings. Intravenous urography showed normal kidneys, while 20 minutes of intravenous urogram of the incompletely filled UB showed a tubular transparency next to the left lateral wall of the UB (Fig. 2). Pathological ultrasonographic and intravenous urography findings indicated cystoscopy, which revealed a thin tube-like structure in the UB, covered with hairs, partly incorporated into the left wall of the bladder, and suspicious of a FB, with surrounding hyperaemia. Tumour mass such as a teratoma could not be excluded, also. As a result and especially due to the fact that the boy denied self-insertion of the $\mathrm{FB}$, cystoscopic removal of the FB could not be performed. The urgent computed tomography (CT) of the pelvis was performed with a 6-row multidetector scanner (MDCT Siemens Emotion). Unenhanced and enhanced CT images revealed a hypodense, tubular area in the UB, spreading caudally through the inflammed left lateral wall of the bladder, into the front part of the left obturator internus muscle (Fig. 3 and 4). That structure was suggestive of to a FB.

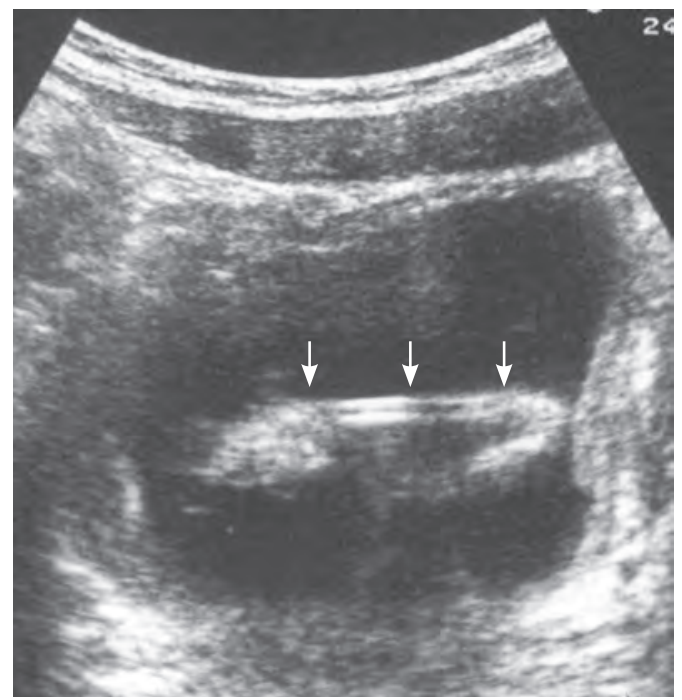

Fig. 1 Ultrasound image shows hyperechogenic tubular structure next to the thickened lateral urinary bladder wall (arrows).

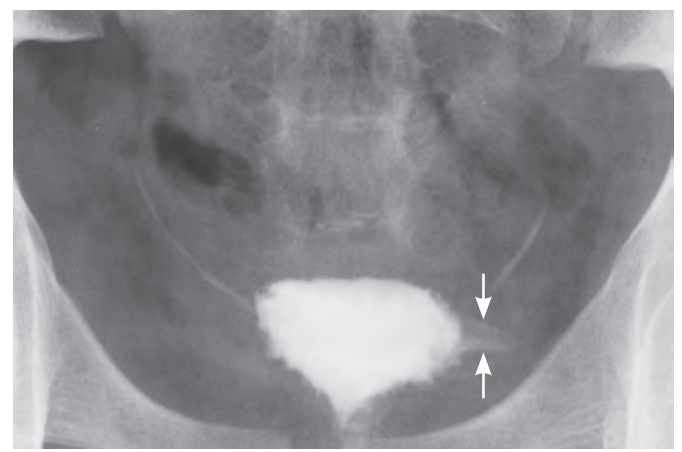

Fig. 220 minutes intravenous urogram shows a tubular transparency boundary marked by contrast next to the left lateral wall of incompletely filled urinary bladder (arows).

Although cystoscopic and radiological findings were indicative for the presence of a FB in the UB, the boy still denied self-insertion of the FB, and the urologist could not exclude a tumour from differential diagnosis. As a result suprapubic cystostomy was done and a yellow-white ear cleaning stick, $10 \mathrm{~cm}$ in length, with a wad on top, was found in the UB, with one end stuck in the hyperaemic and edematous bladder wall. Bladder suture and reconstruction were not necessary. Unfortunately, no photograph was taken of 


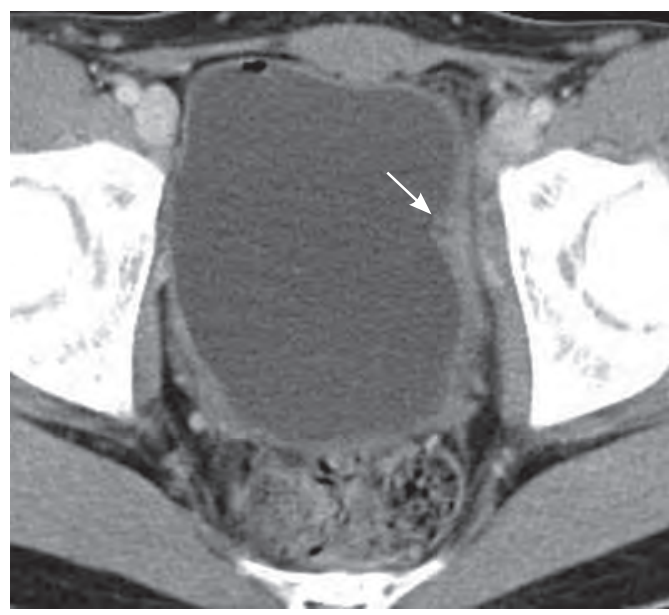

Fig. 3 The axial enhanced CT image shows thickened, inflamed lateral urinary bladder wall on the left with a hypodense, tubular area, partly located into the bladder wall, and partly in the lumen of bladder (black arrows). This area most correspond to foreign body.

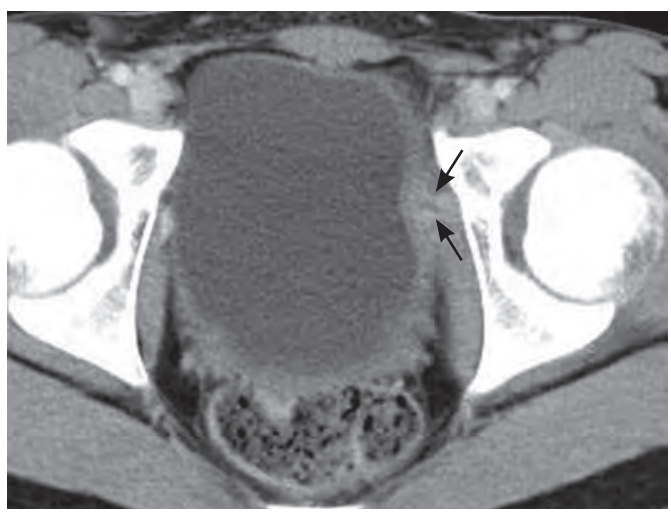

Fig. 4 The axial enhanced CT image of the pelvis shows a hypodense, narrow, tubular area in the front part of the left obturator internus muscle, towards the lateral urinary bladder wall, which most probably corresponds to the foreign body. The perivesical hyperdense area, arround the tubular hypodensity, with contrast enhancement corresponds to inflammatory-granulated tissue.

the FB. The patient recovered successfully and in the end confessed self-insertion of the FB during erotic stimulation. A psychiatric consultation and control examination were suggested but the boy failed to return for his follow up visit.

\section{Discussion}

FBs in the UB, often reported in the literature are an important consideration in the differential diagnosis of lower urinary tract problems. Insertion methods in the bladder include the following: iatrogenic-results of medical errors, migration from adjacent organs, penetrating injury and self-insertion (through the urethra) (5). The incidence of iatrogenic FBs in the UB is on the rise as a result of the large number of surgical procedures being conducted all over the world. Tips of Foley catheters, pieces of balloons, forgotten urological stents, ribbon gauze, clips, encrusted sutures, have all been found in the UB (6-10). In some cases pubic hair may accidentally enter the bladder during catheterization (11). FBs can perforate the UB from the gastrointestinal or female genital tract. Abdominal swabs, gauze, intrauterine contraceptive devices in adult females, bone cement and suture materials have been reported as foreign bodies in the bladder (12).

Trauma, particularly from a bullet, is another causal factor (13). Self-insertion of a $\mathrm{FB}$ via the urethra into the UB was the main mechanism in several reports $(4,14)$. Due to the shorter urethra and its straight alignment, females are more susceptible to ascending migration of FBs than males (14). Almost all items that can pass through the urethra may be found within the bladder (15). Some of the common aetiologies of urethral insertion include psychiatric disorder, autoerotic stimulation and senility (16). Unlike adults where FBs in the bladder are not uncommon, usually during masturbation, self-insertion of FB is rarely seen in children $(2,17)$. In children, intravesical FB are usually inserted at the start of puberty for sexual stimulation $(2,5,14)$. On the other hand, self-insertion in children before puberty is very rare, and the reason for that is almost always inquisitiveness (2). Mannan et al. (7) encountered a four-yearold girl who introduced a hair pin into her 
bladder while playing with it, while Navarro et al. (18) presented a thirteen-year-old boy who introduced a $20 \mathrm{~cm}$ long electric wire into his bladder. Ceran and Uguralp (2) presented two cases of self-inserted urethrovesical FBs in children. Recently, Bhattacharya et al. (19) reported a case of a $8 \mathrm{~cm}$ darning needle stuck in the urethra of a 12-year-old male patient in a suicide attempt.

Patients with intravesical FB sometimes have no symptoms or have minimal discomfort but usually present with dysuria, poor urinary stream or retention, bloody or purulent urethral discharge, urinary tract infection, urgency, and/or pelvic pain (5). Other presenting complaints include voiding difficulties, haematuria, swelling of the genitalia, extravasations of urine, and abscess formation $(5,17)$.

The diagnosis of FB in the UB is a major challenge for urologists and radiologists, and other specialists, such as paediatricians. The physical examination is almost always unremarkable, and urine microscopy usually reveals pus cells and red blood cells (7). Radiopaque urethrovesical objects can be easily visible on plain radiographs. Long-term retention of the FB in the UB may induce stone encrustation (20). Due to encrustation, even un-radiopaque objects may also become apparent on plain radiographs. In the diagnosis of $\mathrm{FB}$, ultrasound has also been employed. The exact location of the FB in the UB, its relationship to surrounding structures, and associated soft tissue injuries can be delineated well on ultrasound $(20,21)$. Cystography involves administration of dilute contrast medium into the bladder under fluoroscopic guidance, and the images obtained during filling, full distension and after drainage may also be helpful. Cystoscopy usually suffices for the diagnosis of the presence and location of the FB, but cystoscopy as well as cystography do not provide any valuable information about the surrounding pelvic structures and the relationship between them and the
FB (23). As a result, in some cases for a diagnosis, it is required to perform $\mathrm{CT}$, as in the present case. CT cystography, where contrast is administered directly into bladder via a Foley catheter and a CT scan is performed subsequently, is highly accurate for diagnosing bladder ruptures. This is particularly significant in the cases of intravesical FB caused by trauma. CT cystography has a sensitivity of $95 \%$ and specificity of $100 \%$ in diagnosing bladder ruptures (23).

Most FBs in the UB can be successfully removed by endoscopy with cystoscopic grasping forceps (7). Laparoscopic techniques for retrieval of $\mathrm{FB}$ from the UB are especially useful in children, where larger scopes cannot be used transurethrally (24). Open removal via suprapubic cystotomy is sometimes required, as in the present case. Complications following these procedures are rare but can include infection, fistula, urethral stricture, diverticulum, and incontinence. Of these, urethral strictures are the most common delayed complication. Thus, appropriate follow-up is essential $(7,25)$.

\section{Conclusion}

In the differential diagnosis of unknown genital bleeding and haematuria, one should always consider a FB, even when the patient denies self-insertion. Early diagnostic procedures, cystoscopy examination and radiological evaluation are necessary to determine the exact size, number, and nature of the FB, as well as its position, its possible insertion into the walls of the bladder or possible perforation of the urinary bladder. Adequate diagnosis of the FB is necessary for selection of the appropriate method of removal of the $\mathrm{FB}$, that must be individualized according to the size and nature of the FB and the age of the patient.

Authors' contributions: Conception and design: SM; Acquisition, analysis and interpretation of data: SM, 
SZ, SJ. Drafting the manuscript: SM; Revising it critically for important intellectual content: SM.

Conflict of interest: The authors declare that they have no conflict of interest.

\section{References}

1. Moskalenko VZ, Litovka VK, Zhurilo IP, Mal'tsev VN, Latyshev KV. Foreign body of bladder in children. Klinicheskaya Khirurgiya. 2002;4:43-5.

2. Ceran C, Uguralp S. Self-Inflicted Urethrovesical Foreign Bodies in Children. Case Reports in Urology. 2012 [cited 2012 Aug 9]. Available from: http://www.ncbi.nlm.nih.gov/pmc/articles/ PMC3423797.

3. Hiller N, Zagal I, Solomom I. Intravesical foreign bodies. The danger of eroticism. Urologia. 1999;66(2):168-9.

4. Kochakarn W, Pummanagura W. Foreign bodies in the female urinary bladder: 20-year experience in Ramathibodi Hospital. Asian J Surg. 2008;31(3):130-3.

5. Rafique M. Intravesical Foreign Bodies Review and Current Management Strategies. Urology Journal. 2008;5:4:223-31.

6. Eckford SD. Intravesical foreign bodies: five years review. BJU Int. 1992;69:41-5.

7. Mannan A, Anwar S, Qayyum A, Tasneem RA. Foreign bodies in the urinary bladder and their managemenet: a Pakistani experience. Singapore Med J. 2011;52(1):24-8.

8. Somers WJ. Management of forgotten or retained indwelling ureteral stents. Urology. 1996;47:4315.

9. Forester ND, Evans C, Thomas DFM, Najmaldin A. Bladder stones associated with synthetic absorbable sutures in children. BJU Int. 2001;88:984.

10. Ward HC. Surgical staples in bladder calculi after caecocystoplasty. Br J Urol. 1987;60:375.

11. Zeitlin AB, Cottrell TL, Lloyd FA. Hair as a lower urinary tract foreign body. J Urol. 1957;77:840.

12. De Gier RP, Feitz WF. Surgical instrument migration from the abdominal cavity through the blad- der into the vagina; a rare long-term complication. Urology. 2002;60:165.

13. Gulanikar A, Pandey P, Terrell F. A bullet in the bladder. BJU Int. 1998;82:304.

14. Van Ophoven A, de Kernion JB. Clinical management of foreign bodies of the genitourinary tract. J Urol. 2000;164:274-87.

15. Abdulla MM. Foreign body in the bladder. BJU. 1990;65:420.

16. Rahman NU, Elliott SP, McAninch JW. Self-inflicted male urethral foreign bodies insertion: endoscopic management and complications. BJU Int. 2004;94:1051-3.

17. Rajesh L, Kader A, Bhat VB. Unusual foreign body in the male urethra. Indian Pediatrics. 2000;37(4):450-2.

18. Navarro HP, Ruiz JM, Sanchiz CM, Teruel MP, Guzman JMP, Sanchez AS, et al. Bladder foreign body. Arch. Esp. Urol. 2011;64(7):643-4.

19. Bhattacharya P, Kar HS, Barman A, Chatterjee S, Saha AK. Suicidal introduction of a darning needle in urethra of a male child: a case report. Journal of Evolution of Medical and Dental Sciences. 2014;3(6):1542-5.

20. Huang YH, Kuo JY, Chen KK, Chiu AW, Chang LS. Bilateral Hydronephrosis Caused by Intravesical Foreign Body: A Case Report. JTUA. 2005;16(1):21-3.

21. Lazar J, Asrani A. Sonographic Diagnosis of a Glass Foreign Body in the Urinary Bladder. J Ultrasound Med. 2004; 23:969-71.

22. Barzilai M, Cohen I, Stein A. Sonographic detection of a foreign body in the urethra and urinary bladder. Urologia Internationalis. 2000;64(3):17880 .

23. Power N, Ryan S, Hamilton P. Computed Tomography cystography in bladder trauma: pictorial essay. Can Assoc Radiol J. 2004;55:304-8.

24. Hutton KAR, Huddart SN. Percutaneous retrieval of an intravesical foreign body using direct transurethral visualisation: a technique applicable to small children. BJU Int. 1999;83:337.

25. Naidu K, Chung A, Mulcahy M. An unusual urethral foreign body. International Journal of Surgery Case Reports. 2013;4(11):1052-54. 